\title{
Increased photorefractive sensitivity in double-doped $\mathrm{KTa}_{1-\mathrm{x}} \mathrm{Nb}_{\mathrm{x}} \mathrm{O}_{3}: \mathrm{Fe}, \mathrm{Ti}$
}

\author{
Victor Leyva, ${ }^{*}$ Aharon Agranat, ${ }^{\dagger}$ and Amnon Yariv \\ Department of Applied Physics, California Institute of Technology, Pasadena, California 91125
}

Received December 28, 1992

\begin{abstract}
Double-doped KTN:Fe,Ti exhibits increased photorefractive sensitivity compared with single-doped KTN:Fe and KTN:Ti. Absorption and photoconductivity measurements correlate the increase with an increased concentration of $\mathrm{Fe}$ in the reduced $\mathrm{Fe}^{2+}$ valence state and to an increased fraction of Fe incorporated into the crystal from the flux.
\end{abstract}

Photorefractive materials have received increased interest as volume holographic storage media. Material limitations, including low photorefractive sensitivity, remain to be overcome. In ferroelectric oxides the photorefractive properties are strongly dependent on the type, concentration, and valency of transition-metal impurities, ${ }^{1}$ all of which can be modified either during or after the crystal growth stage. Dopants added to the flux during the growth stage determine the identity and the total concentration of the photorefractive center. Postgrowth oxidation/reduction treatments modify the valence state of dopant ions through the creation or filling of oxygen vacancies. ${ }^{2,3}$

Both the site occupancy and the valency of dopant ions can also be modified in combination with a double dopant. Electron-paramagnetic-resonance results of Hannon in $\mathrm{KTaO}_{3}$ indicate that, in singledoped samples, $\mathrm{Fe}^{3+}$ substitutionally occupies the $\mathrm{Ta}$ site. By codoping with $\mathrm{Ti}$, the $\mathrm{Fe}^{3+}$ is biased into the $\mathrm{K}$ site. ${ }^{4}$ In $\mathrm{LiNbO}_{3}$ waveguides, $\mathrm{Ti}$ indiffusion leads to a reduction of the valence state of $\mathrm{Fe}$ impurities and to a resultant increase in photorefractive damage. ${ }^{5}$ In this Letter we describe results in which double doping of $\mathrm{KTa}_{1-x} \mathrm{Nb}_{x} \mathrm{O}_{3}$ (KTN) with $\mathrm{Fe}$ and Ti leads to an increased photorefractive sensitivity compared with that of single-doped samples. The increased sensitivity is a result of an increase of $\mathrm{Fe}$ in the reduced $\mathrm{Fe}^{2+}$ valence state as determined from absorption measurements. An increase in the fraction of Fe that enters the crystal from the flux is also observed.

Three KTN crystals with different dopant combinations were grown with the top-seeded solution growth method. ${ }^{6}$ The first crystal was doped with $0.5 \mathrm{~mol} . \%$ of $\mathrm{Fe}$ in the flux, the second with 0.5 mol. \% of Ti, and the third with $0.25 \mathrm{~mol}$. \% of Fe and $0.25 \mathrm{~mol}$. \% of Ti. Samples were cut from each crystal and polished along the [001] crystallographic axes. Metal electrodes were evaporated onto each sample. The KTN:Fe sample measured $5 \mathrm{~mm} \times 5.7 \mathrm{~mm} \times$ $3.7 \mathrm{~mm}$, the KTN:Ti sample measured $6.4 \mathrm{~mm} \times$ $6.7 \mathrm{~mm} \times 2.9 \mathrm{~mm}$, and the KTN:Fe,Ti sample measured $3.8 \mathrm{~mm} \times 3.8 \mathrm{~mm} \times 5.5 \mathrm{~mm}$. The chemical composition of each sample was determined with electron microprobe analysis. The results are sum- marized in Table 1. The Fe and Ti concentrations were below the detection limit of 100 parts in $10^{6}$ by weight. The Curie temperatures were determined from peaks in the dielectric constant to occur at $T_{c}=$ $-8^{\circ} \mathrm{C}$ for the KTN:Fe sample, $T_{c}=-15^{\circ} \mathrm{C}$ for the KTN:Ti sample, and $T_{c}=-28^{\circ} \mathrm{C}$ for the $\mathrm{KTN}$ : Fe,Ti sample. The variations in the Curie temperatures are a result of the differing $\mathrm{Ta} / \mathrm{Nb}$ concentration ratios. $^{7}$

The absorption spectrum of each sample was measured with a double-beam spectrophotometer, and all the spectra are shown in Fig. 1. The double-doped sample exhibits the largest absorption. The KTN:Ti sample has low absorption, which suggests that the absorption in the KTN: $\mathrm{Fe}$, Ti sample is due primarily to the Fe. The difference between the absorption in the KTN:Fe,Ti sample and in the KTN:Fe sample is shown in Fig. 2. Features in the difference spectra should be a result of the dopant ions, with a magnitude proportional to the difference in concentration of the absorbing ions. Two features are visible in the difference spectra. The band centered at $460 \mathrm{~nm}$ $(2.70 \mathrm{eV})$ is identified as that which produces a free electron by photoexcitation of a $\mathrm{Fe}^{2+}$ ion. The width of the band in $0.66 \mathrm{eV}$. The $\mathrm{Fe}^{+2}$ charge-transfer transition is broader and peaked at a lower energy than that determined for $\mathrm{Cu}^{+}$in KTN (peak, 3.0 $\mathrm{eV}$; width, $0.38 \mathrm{eV}$ ). ${ }^{3}$ The other feature visible in the difference spectra is the edge of an absorption band visible at $400 \mathrm{~nm}$. This is identified as the edge of the band that generates a free hole by photoexcitation of the $\mathrm{Fe}^{3+}$ ion. Hole photoconducivity in $\mathrm{LiNbO}_{3}: \mathrm{Fe}$ has similarly been observed to occur near the band edge at energies higher than that for electron conductivity. ${ }^{8}$

The magnitude of the band at $460 \mathrm{~nm}$ in Fig. 2 is proportional to the difference in $\mathrm{Fe}^{2+}$ concentration between the samples. Similarly, the magnitude of the band edge visible at $400 \mathrm{~nm}$ is proportional to the concentration difference of $\mathrm{Fe}^{3+}$. By comparison with the total absorption at these wavelengths, the KTN:Fe,Ti sample is determined to have a factor-of-3.7 increased $\mathrm{Fe}^{2+}$ (filled donor) concentration compared with the KTN:Fe sample. Similarly the KTN:Fe sample is determined to have a factorof-1.3 increased $\mathrm{Fe}^{3+}$ (electron trap) concentration 
Table 1. Material Parameters of the KTN Samples Determined from Absorption and Photoconductivity Measurements

\begin{tabular}{lcccc}
\multicolumn{1}{c}{ Sample } & {$\left[\mathrm{Fe}^{2+}\right]$ (a.u.) } & {$\left[\mathrm{Fe}^{3+}\right]$ (a.u.) } & $\alpha \mu \tau_{R}\left[\mathrm{~cm} /(\mathrm{V} \mathrm{s}), \times 10^{-10}\right]$ & $\mu \tau_{R}\left[\mathrm{~cm}^{2} /(\mathrm{V} \mathrm{s}), \times 10^{-10}\right]$ \\
\hline $\mathrm{KTa}_{0.70} \mathrm{Nb}_{0.30} \mathrm{O}_{3}: \mathrm{Fe}, \mathrm{Ti}$ & 3.7 & 1 & 6.24 & 4.99 \\
$\mathrm{KTa}_{0.68} \mathrm{Nb}_{0.32} \mathrm{O}_{3}: \mathrm{Fe}$ & 1 & 1.3 & 1.02 & 2.72 \\
$\mathrm{KTa}_{0.67} \mathrm{Nb}_{0.33} \mathrm{O}_{3}: \mathrm{Ti}$ & - & - & 0.86 & 1.72 \\
\hline
\end{tabular}

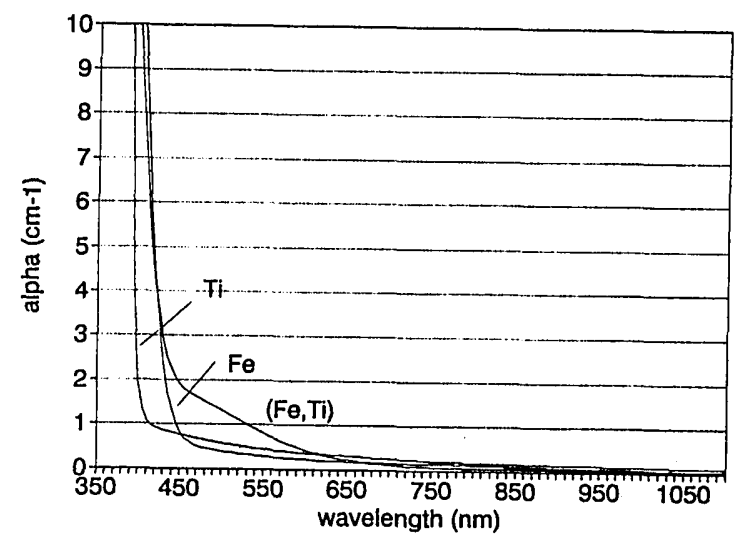

Fig. 1. Absorption spectra of the KTN:Fe,Ti, KTN:Fe, and KTN:Ti samples.

compared with the KTN:Fe,Ti sample. The addition of Ti significantly increases the Fe that enters the crystal in the reduced $\mathrm{Fe}^{2+}$ state. The effect is even more pronounced when one considers that the flux used to grow the KTN:Fe,Ti crystal had half as much $\mathrm{Fe}$ as the flux used to grow the KTN:Fe crystal. In addition, assuming that $\mathrm{Fe}$ enters primarily in the $2+$ and $3+$ valence states, the fraction of $\mathrm{Fe}$ incorporated into the crystal from the flux is increased by codoping with Ti. Transition-metal impurities substitutionally occupy lattice sites in perovskites. The bias of $\mathrm{Fe}$ into the $2+$ valence state when it is codoped with Ti may be a result of charge compensation of the lattice. Compensation occurs when a $\mathrm{Ti}^{4+}$ ion occupies a $(\mathrm{Ta}, \mathrm{Nb})^{5+}$ site and a $\mathrm{Fe}^{2+}$ ion occupies a $\mathrm{K}^{+}$site.

The photocurrent of each sample was measured under 514-nm illumination. Values for the products $\alpha_{\mathrm{ct}} \mu \tau_{R}$ and $\mu \tau_{R}$ determined from the measurements are given in Table 1, where $\alpha_{\mathrm{ct}}$ is the coefficient for absorption that results in a free carrier and is proportional to the concentration of absorbing ions, $\mu$ is the electron mobility, and $\tau_{R}$ is the free-carrier lifetime (recombination time) that is inversely proportional to the trap concentration. ${ }^{3}$ The KTN:Fe,Ti sample exhibits an increased photocurrent compared with the KTN:Fe and KTN:Ti samples, while the mobility lifetime products vary little. The results are consistent with the relative $\mathrm{Fe}^{2+}$ and $\mathrm{Fe}^{3+}$ concentrations determined from the absorption measurements.

Holographic diffraction measurements were made on each sample in the paraelectric cubic phase, which required the application of an external field. Diffraction gratings were written by using two expanded beams from a 514-nm Ar laser. The beams had intensities of 20 and $110 \mathrm{~mW} / \mathrm{cm}^{2}$ (intensity modulation $m=0.2$ ) and were ordinarily polarized in order to limit photorefractive wave coupling. A field of
$1820 \mathrm{~V} / \mathrm{cm}$ was applied to the samples, and the writing beams were adjusted to form a $5.1-\mu \mathrm{m}$ grating period. The diffracted intensity of an extraordinarily polarized weak probe beam from a $\mathrm{He}-\mathrm{Ne}$ laser aligned at the Bragg angle monitored the strength of the grating.

For weak coupling the diffraction efficiency $\eta$ is given by that from a thick plane holographic grating ${ }^{9}$ :

$$
\eta=\exp (-\alpha l) \sin ^{2}\left[\frac{\pi \Delta n}{\lambda \cos (\theta / 2)}\right],
$$

where $\alpha$ is the total absorption, $l$ is the thickness of the sample, $\Delta n$ is the magnitude of the index grating, $\lambda$ is the wavelength of the illuminating radiation, and $\theta$ is the angle between the writing beams. We determined the erase time $\tau$ by monitoring the exponential decay of $\Delta n$ under $110-\mathrm{mW} / \mathrm{cm}^{2}$ illumination after one of the writing beams was blocked. The photorefractive sensitivity, defined as the refractiveindex change per absorbed unit of energy, is given
by $^{10}$

$$
S=\frac{\mathrm{d} n}{\mathrm{~d}(\alpha I t)}=\frac{\Delta n}{\alpha I \tau},
$$

where $I$ is the intensity.

We placed the samples on a thermoelectric mount and measured the refractive-index change and the erase rate at several temperatures above each sample's Curie temperature. Both the diffraction efficiency and the response time increase as the Curie temperature is approached. The photorefractive sensitivity has a much weaker temperature dependence than do $\Delta n$ and $\tau$ near the phase transition, which allows for comparisons among samples with differing transition temperatures. Results for the temperature dependence of the photorefractive

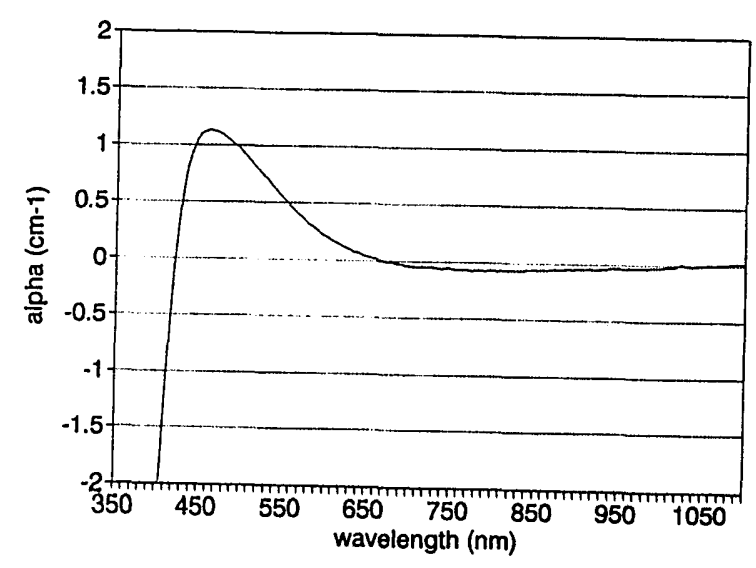

Fig. 2. Difference in the absorption spectra of the $\mathrm{KTN}: \mathrm{Fe}, \mathrm{Ti}$ and KTN:Fe samples. 


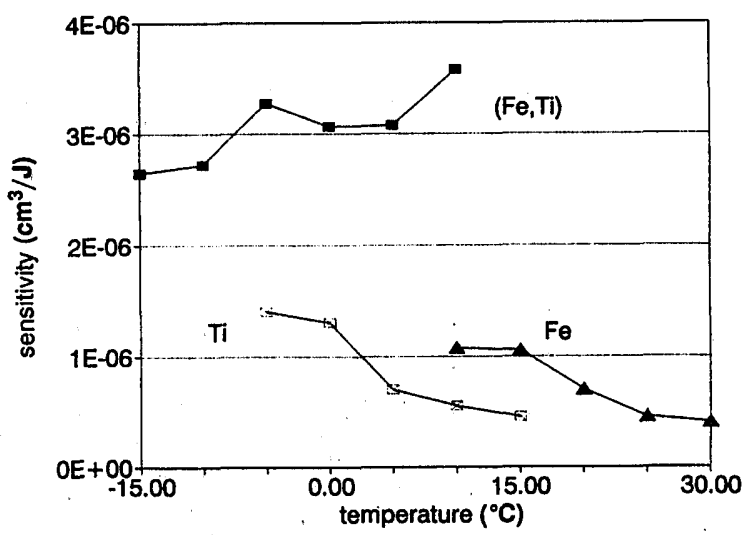

Fig. 3. Photorefractive sensitivity measurements of the KTN:Fe,Ti, KTN:Fe, and KTN:Ti samples above each sample's Curie temperature $(\lambda=514 \mathrm{~nm}, \Lambda=5.1 \mu \mathrm{m}$, $E=1820 \mathrm{~V} / \mathrm{cm}$, and $m=0.2$ ).

sensitivity for each sample are given in Fig. 3. The KTN:Fe,Ti sample exhibits more than a factor-of-3 increased sensitivity compared with the single-doped samples. The higher sensitivity is due mainly to a faster response time for the double-doped sample. The photoconductivity measurements indicate that the faster response is due to a larger absorption from the increased concentration of the $\mathrm{Fe}^{2+}$ ions rather than to an increased free-carrier lifetime. An increased photorefractive sensitivity is also observed in samples in which the photorefractive center is reduced through the creation of oxygen vacancies. ${ }^{3}$ The double-dopant approach described here does not require any postgrowth processing. It can also be applied to other host crystals, including $\mathrm{KNbO}_{3}$, $\mathrm{LiTaO}_{3}$, and $\mathrm{LiNbO}_{3}$, in which the effect has been observed in Ti-indiffused waveguides. ${ }^{5}$
The photorefractive properties of Fe-doped, Tidoped, and Fe- and Ti-double-doped KTN samples were investigated. Absorption measurements indicate that the double-dopant combination of $\mathrm{Fe}$ and Ti increases the fraction of $\mathrm{Fe}$ in the reduced $\mathrm{Fe}^{2+}$ valence state and increases the fraction of $F e$ that enters the crystal from the flux compared with that of single-doped $\mathrm{Fe}$ sample. The double-dopant combination of $\mathrm{Fe}$ and $\mathrm{Ti}$ leads to a factor-of-3 increased photorefractive sensitivity compared with single-doped samples.

The authors acknowledge the support of the U.S. Army Research Office.

${ }^{*}$ Present address, Accuwave Corporation, 1653 19th Street, Santa Monica, California 90404.

${ }^{+}$Present address, Division of Applied Physics, Hebrew University, Jerusalem 91904, Israel.

\section{References}

1. W. Phillips, J. J. Amodei, and D. L. Staebler, RCA Rev. 33, 94 (1972).

2. W. Phillips and D. L. Staebler, J. Electron. Mater. 3, 610 (1974).

3. V. Leyva, A. Agranat, and A. Yariv, J. Opt. Soc. Am. B 8, 701 (1991).

4. D. M. Hannon, Phys. Rev. 164, 164 (1967).

5. P. Gericke, P. Hertel, E. Kratzig, J. P. Nisius, and R. Sommerfeldt, Appl. Phys. B 44, 155 (1987).

6. A. Agranat, V. Leyva, K. Sayano, and A. Yariv, Proc. Soc. Photo-Opt. Instrum. Eng. 1148, 52 (1989).

7. S. Treibwasser, Phys. Rev. 114, 63 (1959).

8. E. Kratzig and R. Orlowski, Ferroelectrics 27, 241 (1980).

9. H. Kogelnik, Bell Syst. Tech. J. 48, 2909 (1969).

10. G. C. Valley and M. B. Klein, Opt. Eng. 22, 704 (1983). 